\title{
Retrospective validation of whole genome sequencing- enhanced surveillance of listeriosis in Europe, 2010 to 2015
}

Ivo Van Walleㄹ, Jonas Torgny Björkman², Martin Cormican³, Timothy Dallman4, Joël Mossong ${ }^{5}$, Alexandra Moura6, Ariane Pietzka7, Werner Ruppitsch ${ }^{7}$, Johanna Takkinen ${ }^{1}$, European Listeria WGS typing group ${ }^{8}$

1. European Centre for Disease Prevention and Control (ECDC), Stockholm, Sweden

2. Statens Serum Institut, Copenhagen, Denmark

3. National University of Ireland Galway, Galway, Ireland

4. Public Health England, London, United Kingdom

5. Laboratoire national de santé, Dudelange, Luxembourg

6. Institut Pasteur, National Reference Center and WHO Collaborating Center Listeria, Biology of Infection Unit, Inserm U1117, Paris, France

7. Österreichische Agentur für Gesundheit und Ernährungssicherheit, Graz/Vienna, Austria

8. The European Listeria WGS typing group members have been listed at the end of this article

Correspondence: Ivo van Walle (ivo.van.walle@ecdc.europa.eu)

Citation style for this article:

Van Walle Ivo, Björkman Jonas Torgny, Cormican Martin, Dallman Timothy, Mossong Joël, Moura Alexandra, Pietzka Ariane, Ruppitsch Werner, Takkinen Johanna, European Listeria WGS typing group. Retrospective validation of whole genome sequencing-enhanced surveillance of listeriosis in Europe, 2010 to 2015 . Euro Surveill. 2018;23(33):pii=1700798. https://doi.org/10.2807/1560-7917.ES.2018.23.33.1700798

Background and aim: The trend in reported case counts of invasive Listeria monocytogenes $(\mathrm{Lm})$, a potentially severe food-borne disease, has been increasing since 2008. In 2015, 2,224 cases were reported in the European Union/European Economic Area (EU/ EEA). We aimed to validate the microbiological and epidemiological aspects of an envisaged EU/EEAwide surveillance system enhanced by routine whole genome sequencing (WGS). Methods: WGS and core genome multilocus sequence typing (cgMLST) were performed on isolates from 2,726 cases from $27 \mathrm{EU} /$ EEA countries from 2010-15. Results: Quality controls for contamination, mixed $\mathrm{Lm}$ cultures and sequence quality classified nearly all isolates with a minimum average coverage of the genome of $55 \mathrm{x}$ as acceptable for analysis. Assessment of the cgMLST variation between six different pipelines revealed slightly less variation associated with assembly-based analysis compared to reads-based analysis. Epidemiological concordance, based on 152 isolates from 19 confirmed outbreaks and a cluster cutoff of seven allelic differences, was good (sensitivity > $95 \%$ for two cgMLST schemes of 1,748 and 1,701 loci each; PPV 58-68\%). The proportion of sporadic cases was slightly below $50 \%$. Of remaining isolates, around one third were in clusters involving more than one country, often spanning several years. Detection of multi-country clusters was on average several months earlier when pooling the data at EU/EEA level, compared with first detection at national level. Conclusions: These findings provide a good basis for comprehensive EU/EEA-wide, WGS-enhanced surveillance of listeriosis. Time limits should not be used for hypothesis generation during outbreak investigations, but should be for analytical studies.

\section{Introduction}

Invasive infection by Listeria monocytogenes $(L m)$ leads to relatively rare but serious food-borne disease mainly affecting elderly people, immunocompromised individuals and pregnant women. Clinical manifestations include sepsis and infection of the central nervous system, which can lead to lifelong sequelae or death $[1,2]$. Pregnancy-associated listeriosis can result in preterm birth, miscarriage or stillbirth [2,3]. In the European Union and European Economic Area (EU/EEA), 2,224 human cases of invasive listeriosis were reported in 2015 , with an overall case fatality rate of $18.8 \%$ [4]. Reported numbers of cases of listeriosis suggest that the incidence of disease slightly increased over the period of 2010-15. The incubation period of listeriosis is usually 3 to 21 days, but can be as long as 67 days, depending on the clinical form of the disease [5]. Patients frequently have underlying conditions and/ or are elderly, which limits the collection of exposure data in some cases. On epidemiological grounds, most cases are considered sporadic and detected outbreaks usually involve small numbers of patients, which limits statistical power in analytical epidemiological studies. As a result, most reported cases of listeriosis are difficult to link to a specific food product or food business operator. 


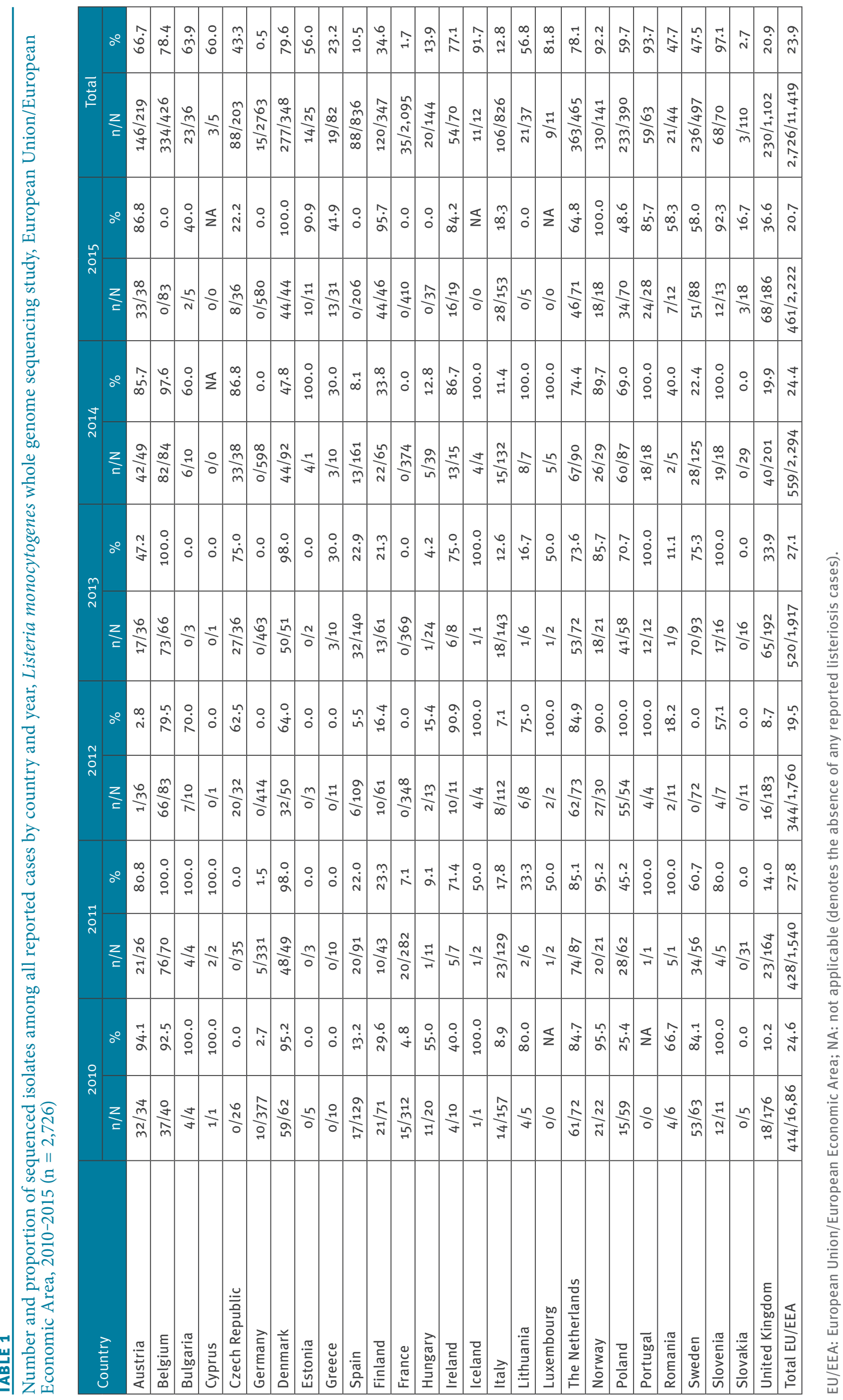


Sequence data quality control based on core genome coverage (CGC), (A) Impact of decreasing CGC on allelic distance ( $\mathrm{n}=$ 2,664), (B) Impact of average coverage, before and after trimming, on passing the CGC quality control, using rarefaction ${ }^{\text {a }}$ (n

$=2,609)$, Listeria monocytogenes whole genome sequencing study, European Union/European Economic Area, 2010-2015

A. Impact of decreasing CGC on allelic distance

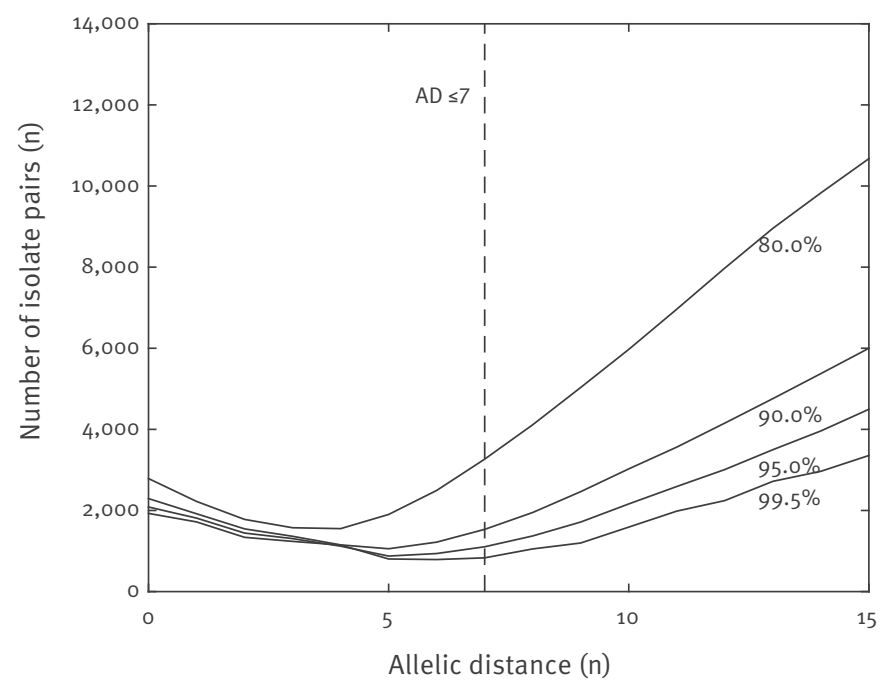

B. Impact of average coverage, before and after trimming, on passing the CGC QC, using rarefaction ${ }^{\mathrm{a}}$

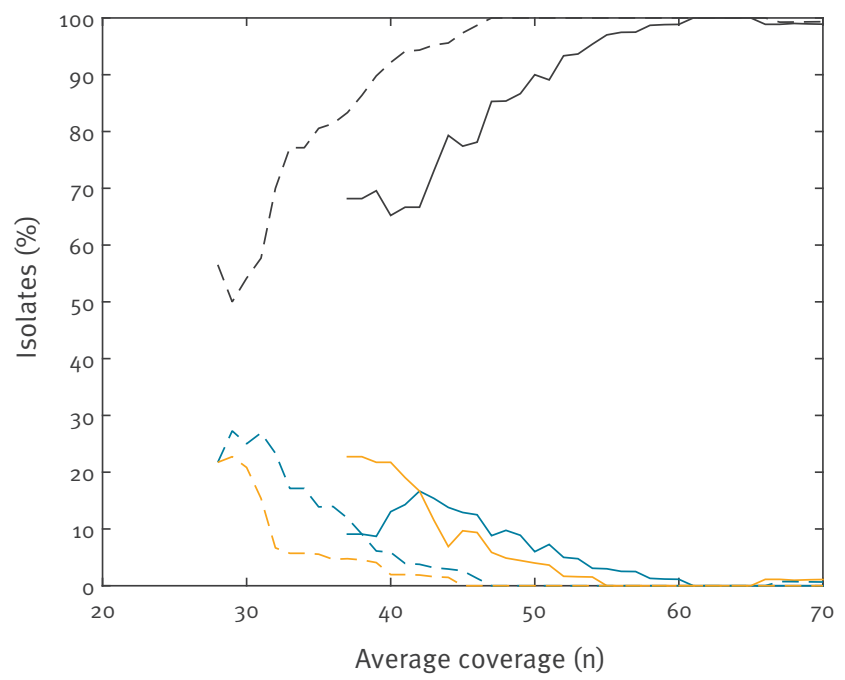

CGC PASS, before trimming

- CGC WARN, before trimming
--- CGC WARN, after trimming

CGC FAIL, before trimming

- - - CGC PASS, after trimming

--- CGC FAIL, after trimming

AD: allelic distance; CGC: core genome coverage; QC: quality control.

${ }^{\mathrm{a}} \mathrm{A}$ sliding window of $\pm 5 \mathrm{x}$ coverage was used, with a minimum number of 10 observations per window.

$L m$ is able to form biofilms, grow at refrigeration temperature, high salt and nitrite concentrations, and can be resistant to disinfectants $[6,7]$. These properties contribute to its ability to persist and multiply in the food-processing environment and make it difficult to control. In the United States (US), a nationwide subtyping of $L m$ using pulsed-field gel electrophoresis (PFGE) was introduced in 1998. During the following 6 years, there was a more than fivefold increase in the number of outbreaks where a common food vehicle could be identified [8]. In the subsequent 10 years, the introduction of detailed food history gathering for all listeriosis patients resulted in a further increase in the number of solved outbreaks per year, as well as a reduction of the number of cases per outbreak [9].

PFGE is, however, time-consuming and difficult to standardise. In recent years, it has been demonstrated that whole genome sequence (WGS)-based subtyping can provide substantial additional discrimination and, consequently, can be of benefit to outbreak investigations [9-11]. Within the EU/EEA, listeriosis is one of the priority diseases for which supranational WGS-enhanced surveillance will be initiated in 2018 [12]. The work presented here supports the preparation for this surveillance system through a large-scale, retrospective, multi-centre study on $L m$ isolates from human cases from EU/EEA countries by covering the comparison and validation of analytical pipelines, the assessment of the epidemiological concordance of the results and the potential impact on public health [13]. The analytical pipelines are based on the gene-by-gene approach recommended by the PulseNet International global consortium [14-17].

\section{Methods}

WGS was performed on a total of 2,726 Lm isolates from human cases from $27 \mathrm{EU} / \mathrm{EEA}$ countries and spanning years 2010-15 using Illumina MiSeq (Illumina, Inc., San Diego, California, US) 2x150 $(n=295)$, Illumina MiSeq $2 \times 250(n=243)$, Illumina MiSeq $2 \times 300(n=98)$, Illumina NextSeq $2 \times 150(n=1,585)$ and Illumina HiSeq $2 \times 100(n=422)$, as well as lonTorrent PGM (Thermo Fisher Scientific, Waltham, Maryland, US) $(n=83)$ platforms. Ten EU/EEA national public health laboratories $(n=1,069$ isolates) and a commercial sequencing pro$\operatorname{vider}(n=1,657$ isolates) performed the sequencing.

Trimming and de novo assembly

Trimming was performed on Illumina reads with Trimmomatic, before any further analysis. This included (i) removal of any adaptor sequences, (ii) removal of 
Independent contribution of source data and scheme to variation in allelic distance between different allele calling methods, Listeria monocytogenes whole genome sequencing study, European Union/European Economic Area, 2010-2015

\begin{tabular}{|c|c|c|c|c|c|c|c|}
\hline \multirow{2}{*}{ Method 1} & \multirow{2}{*}{ Method 2} & \multirow{2}{*}{$\begin{array}{l}\text { Pairs for comparison } \\
A D \leq 7 \text { (n) vs } A D \leq 150 \text { (n) }\end{array}$} & \multirow{2}{*}{$\begin{array}{l}\text { Mean CGC } \\
\text { comparison } \\
\text { method 1 (\%) } \\
\text { vs } 2(\%)\end{array}$} & \multicolumn{2}{|c|}{$\Delta_{A D}{ }^{a}$, subset $A D \leq 7$} & \multicolumn{2}{|c|}{$\begin{array}{l}\Delta_{\mathrm{AD}}{ }^{\mathrm{a}} \text {, subset } \\
\mathrm{AD} \leq 150\end{array}$} \\
\hline & & & & Mean & Range $^{\mathrm{b}}$ & Mean & Range $^{a}$ \\
\hline SPAdes + Moura CG & Velvet + Moura CG & 313 vs 24,196 & 99.5 vs 99.7 & -0.2 & -2 to 0 & -0.8 & -5 to 1 \\
\hline SPAdes + Ruppitsch CG & Velvet + Ruppitsch CG & 1,229 vs 50,233 & 99.2 vs 99.4 & -0.3 & -2 to 0 & -0.6 & -4 to 1 \\
\hline SPAdes + Moura CG & Reads + Moura CG & 2,780 vs 135,365 & 99.5 vs 99.1 & -1.1 & -5 to 1 & -0.6 & -4 to 3 \\
\hline SPAdes + Ruppitsch CG & Reads + Ruppitsch CG & 1,237 VS 50,881 & 99.2 vs 98.9 & -1.2 & -4 to 0 & -1.1 & -5 to 2 \\
\hline Velvet + Moura CG & Reads + Moura CG & 148 vs 9,040 & 99.7 vs 99.1 & -0.6 & -2 to 1 & -0.7 & -5 to 5 \\
\hline Velvet+Ruppitsch CG & Reads + Ruppitsch CG & 1,193 vs 50,381 & 99.4 vs 98.9 & -0.9 & -4 to 1 & -0.6 & -4 to 2 \\
\hline SPAdes + Moura CG & SPAdes + Ruppitsch CG & 5,255 vs 230,478 & 99.5 vs 99.2 & 0.6 & -5 to 3 & -1.9 & -14 to 9 \\
\hline Velvet + Moura CG & Velvet + Ruppitsch CG & 337 vs 24,196 & 99.7 vs 99.4 & -0.0 & -4 to 4 & -1.5 & -12 to 8 \\
\hline Reads + Moura CG & Reads + Ruppitsch CG & 549 vs 19,721 & 99.1 vs 98.9 & 0.2 & -3 to 4 & -0.8 & -13 to 10 \\
\hline
\end{tabular}

AD: allelic distance; CG: core genome; CGC: core genome coverage.

${ }^{\mathrm{a}} \Delta_{\mathrm{AD}}$ is calculated as $A D$ of method 1 minus that of method 2

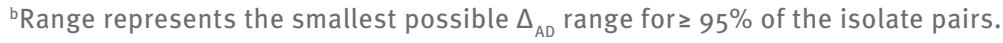

leading bases with PHRED< 25 (i.e.< $99.7 \%$ base call accuracy), (iii) removal of trailing bases with PHRED $<25$, (iv) clipping of the remainder of the read when a sliding window of 20 bases has average PHRED $<25$, and $(v)$ removal of the entire read if length< 36 bases [18].

De novo assembly was performed with SPAdes and Velvet algorithms $[19,20]$. Spades 3.7 .1 was run with BayesHammer read error correction and assembling mode with automatic determination of coverage cutoff, a minimum contig length of 300 nucleotides (nt) and with MismatchCorrector on. BWA-mem 0.7.12 was subsequently used to map all reads back to the SPAdes assembly, and consensus base calling was performed on the resulting alignment as an additional mismatch correction [21]. Velvet 1.1.04 was run with an automatic determination of the coverage cutoff, a minimum contig length of 300 bases using k-mers ranging from 59 to $69 \%$ of the average read length. The assembly with the highest $\mathrm{N}_{50}$ was retained. Bowtie 2 was used to map all reads to this assembly, and consensus base calling was performed on the resulting alignment as mismatch correction [22].

\section{Allele calling}

Allele calling was performed using two core genome multilocus sequence typing (cgMLST) schemes: the scheme of Moura et al. [10], as implemented in the BioNumerics (Applied Maths, Sint-Martens-Latem, Belgium) software (1,748 loci, available from http:// bigsdb.pasteur.fr/listeria) and the scheme of Ruppitsch et al. [23], as implemented in the SeqSphere + (RIDOM, Münster, Germany) software (1,701 loci, available from http://www.cgmlst.org/ncs/schema/690488/). These schemes are further referred to as Moura CG and Ruppitsch CG. Both software applications were run with default parameters for allele calling, both based on either assemblies (assembly-based) or directly on reads (reads-based). In turn, each uses the basic local alignment search tool nucleotide (BLASTN) software to align assembled genomes to reference alleles: word size was 11, gap opening penalty 5 and gap extension penalty 2 [24]. Mismatch penalty was -3 and -1 , and match reward 2 and 1 in Bionumerics 7.6.2 and SeqSphere+3.4.1, respectively. When more than one locus on the assembled genome matches a reference allele, such multiple allele calls are recorded, but no identifier is assigned. The direct reads-based allele calling in BioNumerics matches k-mers of size 35 from all known alleles against those of the reads, and considers the allele found if all its k-mers are present with the same number of occurrences. The SeqSphere + readsbased allele calling first maps the reads to reference genome NC_003210.1 using the BWA-SW software, and then produces a consensus sequence [21]. BLAST is subsequently used to align this sequence against the reference alleles for each locus, keeping only alignments that cover $>90 \%$ of the reference allele [24]. Alignments were verified for presence of start and stop codons and for a minimum of $70 \%$ (Moura CG) or $90 \%$ (Ruppitsch CG) nt sequence identity to reference alleles. For IonTorrent sequences, allele calling was only done by mapping reads first to the reference alleles and then again to the resulting consensus allele sequence, each time removing indels of 1 and 2 nt to specifically address this type of sequencing error.

To assess the impact of the different pipelines, i.e. combinations of input data (directly reads, SPAdes assembly or Velvet assembly) and scheme (Moura CG or Ruppitsch CG) on cgMLST analysis, two subsets of isolate pairs were used: pairs with $A D \leq 7$ and pairs with $A D \leq 150$. These correspond respectively to closely related isolates likely to share a common 


\section{FIGURE 2}

Concordance between cgMLST and epidemiological results for (A) Epidemiologically linked vs unknown or not epidemiologically linked pairs of isolates ${ }^{\mathrm{a}}$, (B) Positive predictive value and sensitivity of single-linkage clustering on confirmed outbreaks, (C) Rarefaction curves for sporadic cases, single-country clusters and multi-country clusters ${ }^{\mathrm{b}}$, (D) Aggregated time span and evolution of clusters'; Listeria monocytogenes whole genome sequencing study European Union/ European Economic Area, 2010-2015 (n = 2,664)

\section{A. Epidemiologically linked vs unknown or not epidemiologically linked pairs of isolates}

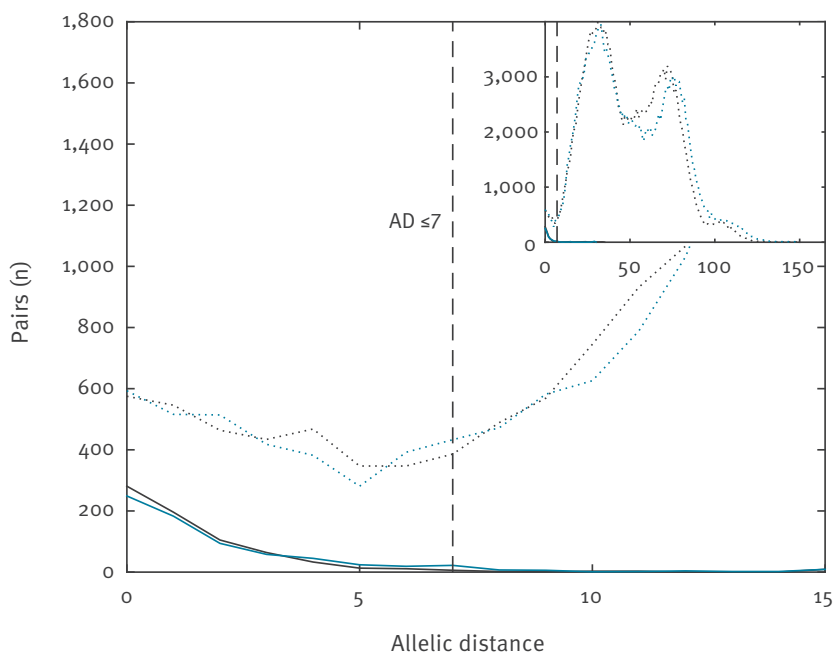

Moura [10], epi linked _ Ruppitsch [16], epi linked …..... Moura [10] , no/unk link …..... Ruppitsch [16], no/unk link

C. Rarefaction curves for sporadic cases, single-country clusters and multi-country clusters ${ }^{\mathrm{b}}$

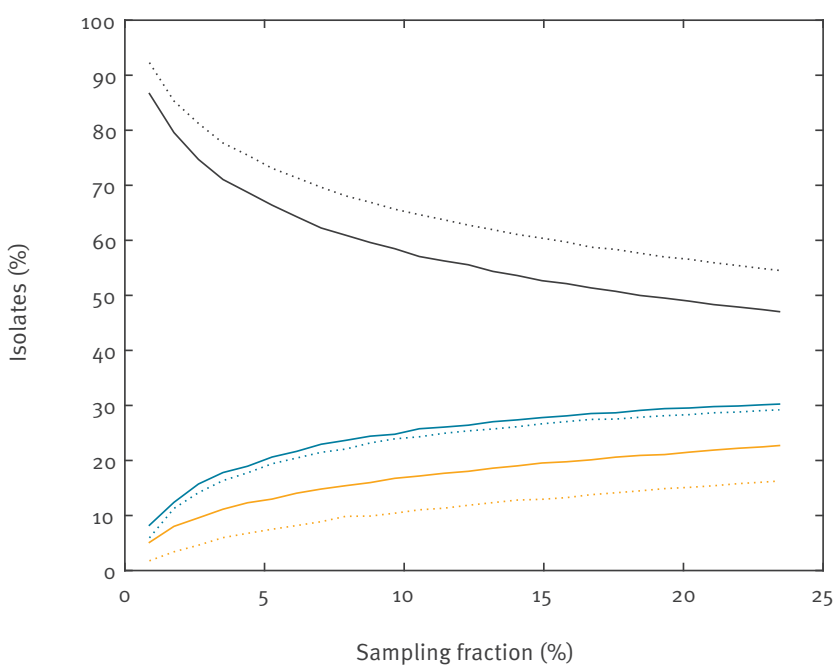

B. PPV and sensitivity of single-linkage clustering on confirmed outbreaks

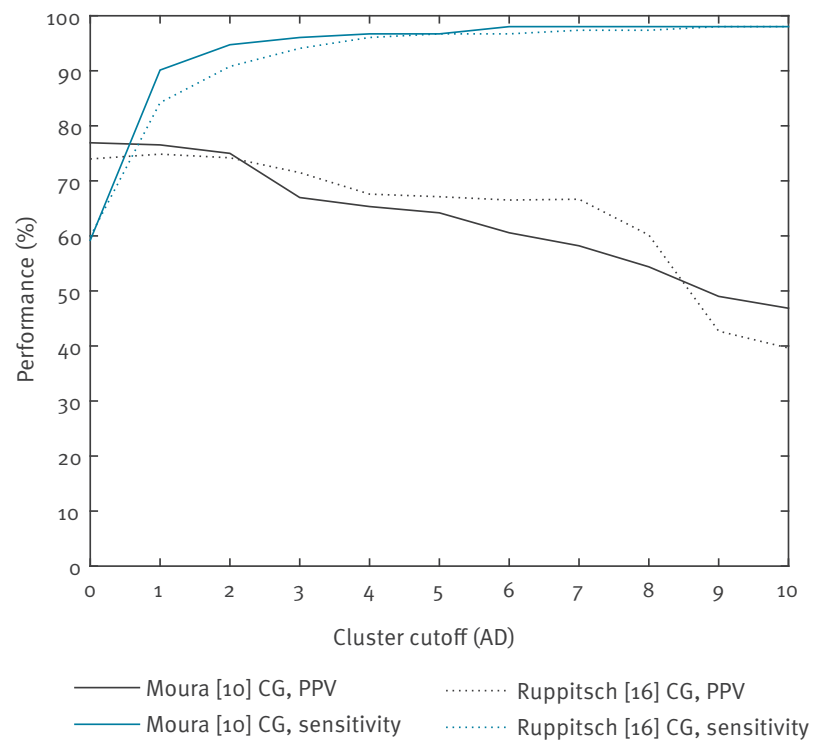

\section{Aggregated time span and evolution of clusters ${ }^{c}$}

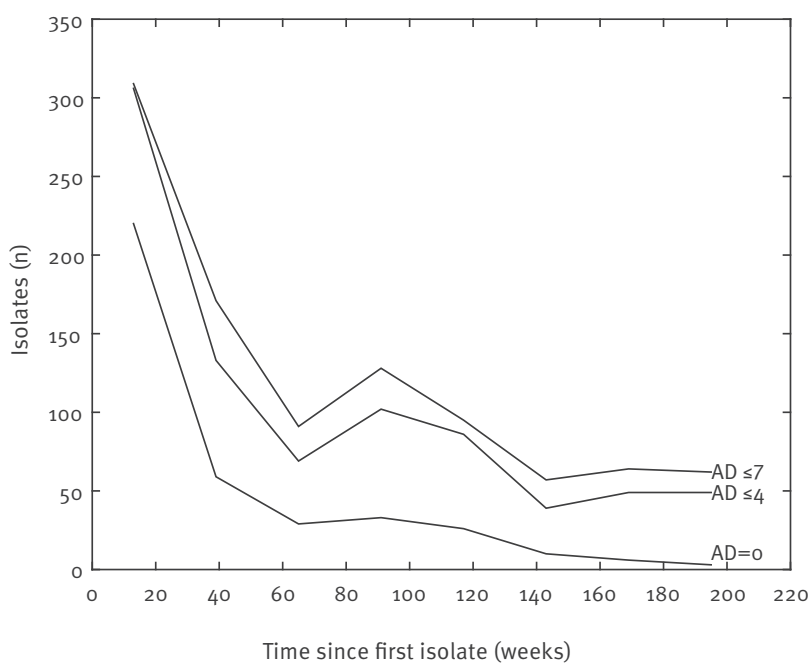

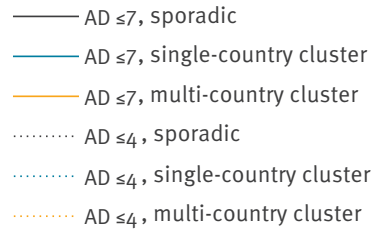

AD: allelic distance; CG: core genome; epi link: epidemiologically linked; no/unk link: unknown or not epidemiologically linked; PPV: positive predictive value.

aThe inset shows all pairs up to $A D \leq 150$.

${ }^{b}$ Cutoff $A D \leq 7$.

${ }^{c}$ Aggregated by setting the first isolate of each cluster at $t=0$ and using bins of 26 weeks, i.e. half a year. 
Single- and multi-country clusters, Listeria monocytogenes whole genome sequencing study, European Union/European Economic Area, 2010-2015

\begin{tabular}{|c|c|c|c|c|c|c|c|c|}
\hline \multirow{3}{*}{$\begin{array}{l}\text { Cluster size } \\
\text { (n) }\end{array}$} & \multirow{3}{*}{ Total (n) } & \multicolumn{2}{|c|}{ Single country } & \multicolumn{5}{|c|}{ Multi-country } \\
\hline & & \multirow[t]{2}{*}{$\mathrm{N}$} & \multirow[t]{2}{*}{$\%$} & \multicolumn{2}{|c|}{$\begin{array}{l}\text { Each country one } \\
\text { isolate }\end{array}$} & \multicolumn{2}{|c|}{$\begin{array}{l}\text { Some countries with more } \\
\text { than one isolate }\end{array}$} & \multirow{2}{*}{$\begin{array}{l}\text { Average time gained through } \\
\text { detection at EU-level (days) }\end{array}$} \\
\hline & & & & $\mathrm{N}$ & $\%$ & $\mathrm{~N}$ & $\%$ & \\
\hline \multicolumn{9}{|c|}{ Cutoff $(A D) \leq 4(n=317)$} \\
\hline 2 & 172 & 132 & 76.7 & 40 & 23.3 & NA & NA & NA \\
\hline $3-5$ & 98 & 60 & 61.2 & 4 & 4.1 & 34 & 34.7 & 144 \\
\hline$>5$ & 47 & 25 & 53.2 & 0 & 0.0 & 22 & 46.8 & 75 \\
\hline \multicolumn{9}{|c|}{ Cutoff $(A D) \leq 7(n=331)$} \\
\hline 2 & 166 & 125 & $75 \cdot 3$ & 41 & 24.7 & NA & NA & NA \\
\hline $3-5$ & 109 & 68 & 62.4 & 4 & 3.7 & 37 & 33.9 & 136 \\
\hline$>5$ & 56 & 25 & 44.6 & 0 & 0.0 & 31 & 55.4 & 154 \\
\hline
\end{tabular}

AD: allelic distance; EU: European Union; NA: not applicable.

epidemiological link, and to sublineages where isolates are still likely to have common phenotypic properties that may be relevant, e.g. for source attribution $[10,25]$.

\section{Quality control}

Numeric quality indicators were defined to assess the following quality issues: contamination, $L m$ mixed cultures and sequence read quality. Two thresholds were determined per indicator to classify the result of the quality control (QC) as (i) acceptable 'PASS' or acceptable with a warning 'WARN' and (ii) not acceptable 'FAIL'. The intermediate WARN level was added for practical application in public health, where a sequence may still be included if the time to resequence is too long compared with the time frame within which action should be taken. A final classification combining the results from all these quality controls is then made: 'Accepted' isolates have all QCS PASS, 'AcceptedForOutbreak' have some QCS WARN but none FAIL and 'Rejected' have at least one QC FAIL.

Contamination checks were performed using BLASTN by aligning the assembled genomes against fully closed genomes of other Listeria species (L. ivanovii, L. marthii, L. seeligeri and L. welshimeri) and species often encountered in laboratories for enteric diseases, including Bacillus cereus, Campylobacter coli, C. fetus, C. hyointestinalis, C. jejuni, C. lari, C. upsaliensis, Clostridium botulinum, $\mathrm{Cl}$. perfringens, Escherichia albertii, E. coli, E. fergusonii, Salmonella enterica, Shigella boydii, S. dysenteriae, S. flexneri, S. sonnei, Vibrio parahaemolyticus and Yersinia enterocolitica [24]. Hits to the reference genome with at least $70 \%$ nt identity were kept and used to determine the proportion of the query genome covered by the reference genome. Mean and standard deviations (SD) of these proportions per reference genome were determined after one round of removing outliers with z-scores higher than 3 for the same genus and $z>6$ for different genus references. Assemblies that were longer than $3.3 \mathrm{Mb}$ were considered to be contaminated with another species as well. The contamination (CNTM) QC was set to WARN when a species of another genus was detected and to FAIL when other Listeria species were detected, since in the latter case it is likely that this will substantially interfere with allele calling.

Checks for $L m$ mixed cultures are much more difficult to assess than contamination with other species due to the inherent similarity between the strains. The $Q C$ used here was based on the multiple allele call results of cgMLST allele calling on SPAdes assembled genomes (see allele calling section). The number of core genome loci with multiple alleles (CGM) was used as an indicator that sequences are likely to originate from contaminated cultures. The CGM QC was set to WARN for isolates with $\mathrm{CGM}=1$, i.e. one locus with multiple allele calls, and to FAIL if CGM > 1 .

Checks for sequence read quality were done based on the core genome coverage (CGC). This was defined as the proportion of core genome loci that were retrieved through allele calling. For each such locus, a biologically meaningful allele can therefore be found, including a start and a stop codon and a minimum similarity to curated reference alleles. Since DNA or sequencing quality issues are not expected to be biased towards core or accessory genome, the CGC values can be expected to be representative of the quality of the whole genome.

\section{Rarefaction analysis}

Rarefaction curves were estimated for the effect of decreasing sampling fraction on both proportion of clustering vs sporadic isolates. This was also done to estimate the effect of decreasing CGC on allelic distances between closely related isolates, in order to 
assess to what extent CGC affects CgMLST analysis and cluster detection. The allelic distance (AD) between a pair of isolates was defined as the number of alleles across all loci in the scheme that are different, ignoring loci not present in either or both isolates. Estimations for all curves were performed with at least 100 random samples per point.

\section{Results}

\section{Dataset}

Table 1 presents the sequenced isolates by country and the respective proportion of officially reported listeriosis cases by year, retrieved from the European Centre for Disease Prevention and Control (ECDC) Surveillance Atlas of Infectious Diseases [4]. Overall in EU/EEA countries, $20-28 \%$ of all isolates from reported cases were sequenced for each year. This is a relatively uniform representativeness, though there was substantial variation between individual countries and also for some countries over the years.

\section{Sequence data quality}

The contamination (CNTM) QC detected three $L$. ivanovii isolates (0.1\%; CNTM result set to FAIL) and 31 isolates that contained bacterial DNA from genera other than Listeria spp. (1.1\%; CNTM set to WARN). The mixed $L m$ culture (CGM) QC detected two isolates with $C G M=1$ (WARN) and six with $C G M>1$ (ranging 2-21; FAIL). Of these eight isolates, five also had CNTM WARN, due to assembly lengths> $3.3 \mathrm{Mb}$.

The final quality issue that was investigated was poor quality of the sequence reads using the CGC QC. Rarefaction analysis of artificially reduced CGC by randomly deleting loci was performed using Moura CG, SPAdes assembly and including only isolates with actual CGC $\geq 95 \%$, CNTM PASS and CGM PASS $(n=2,664)$. This showed that a reduction from $99.5 \%$ on average to $95.0 \%, 90.0 \%$ and $80.0 \%$ led to an increase in the number of isolate pairs with $A D \leq 7$ by $9.9 \%, 26.1 \%$ and $89.5 \%$, respectively (Figure 1 a). Based on this, and the fact that most of these additional pairs remained in the AD $5-7$ region, we selected $\mathrm{PASS} \geq 95 \%$ and $\mathrm{FAIL}<90 \%$ (WARN between 90-95\%) as thresholds for this QC. A total of 2,692 (98.8\%) isolates passed, 16 (0.6\%) passed with a warning and 18 (0.7\%) failed.

Figure $1 b$ shows the impact of the average coverage on the CGC QC, with a clear improvement in the proportion of acceptable isolates as coverage increases up to around $55 \mathrm{x}$ before trimming and $45 \mathrm{x}$ after trimming. Only CNTM PASS and CGM PASS isolates were included and 83 IonTorrent sequences (3.0\%) were excluded from this analysis in order to not mix results from two different platforms, leaving $n=2,609$ isolates for the analysis. In contrast, the corresponding curve for a stricter threshold of $97.5 \%$ for acceptable CGC reaches this plateau only at around $80 x$ coverage before trimming (data not shown).
In summary, for our dataset, 22 isolates (0.8\%) failed at least one QC and were classified as 'Rejected'. No association was found between the sequencing platform used and failing the CGC QC that assesses sequence quality. A total of 40 isolates (1.5\%) passed at least one $\mathrm{QC}$ with a warning but failed none, and these were classified as 'AcceptedForOutbreak'. The remaining 2,664 (97.7\%) passed all three QCs and were classified as 'Accepted', and only these were used for subsequent analyses on analytical and epidemiological validation.

\section{Analytical validation}

The variation introduced by using different input data (directly reads, SPAdes assemblies or Velvet assemblies) and different schemes (Moura CG or Ruppitsch CG) is summarised in Table 2, based primarily on the difference in $A D\left(\Delta_{A D}\right)$, between the most relevant pairs of methods. Other pairs gave near-identical results (data not shown). The $\Delta_{A D}$ between SPAdes and Velvet was small, with a mean of -0.19 vs -0.29 depending on the scheme for $A D \leq 7$. Velvet had in 4,477 of 50,083 (8.9\%) pairs at least one additional allelic difference compared with SPAdes among loci present in both assemblies for the AD $\leq 150$ set, with 83 (0.2\%) for the reverse case. The Velvet assembly length was also shorter in 667 of $1,023(65.2 \%)$ cases where a Velvet assembly was performed. Further assembly-based analyses were therefore based on SPAdes, though results were near-equivalent. The $\Delta_{A D}$ between SPAdesbased and reads-based allele calling was slightly skewed towards more differences based on reads, even though less loci were detected from the reads. Reads-based allele calling therefore seems prone to more variation than assembly-based allele calling. The difference between the two core genome schemes was larger than that between reads and assembly-based allele calling.

\section{Epidemiological validation}

Our dataset contained 19 confirmed outbreaks from 10 EU/EEA countries with a median of 6.5 cases (range: 3-19), plus two mother-child pairs, encompassing 152 isolates. These outbreaks were both microbiologically and epidemiologically confirmed by the respective national authorities, but confirmation was done using microbiological typing methods other than WGS. The outbreaks therefore represent an independent test set for evaluating epidemiological concordance of WGS. The number of corresponding epidemiologically linked pairs decreased with increasing AD (Figure $2 a)$, confirming that low CgMLST AD strongly correlates with epidemiological linkage. This pattern was similar between the two cgMLST schemes, as well as between allele calling approaches (data not shown).

Cluster detection was done using single linkage clustering and subsequently applying a cluster cutoff to the tree. For each of the confirmed outbreaks, only the cluster that had the most isolates in common with the outbreak was considered to be the predicted outbreak. 
Based on this, true positive, false positive and false negative isolates were enumerated and summed across the outbreaks. Figure $2 \mathrm{~b}$ shows both the resulting positive predictive value (PPV) and the sensitivity as a function of the cluster cutoff. As for isolate pairs, both cgMLST schemes have similar performance and, in both cases, sensitivity reaches a plateau of $>95 \%$ at $A D \leq 4$. PPV was $65.3 \%$ vs $67.6 \%$ for Moura CG vs Ruppitsch CG at $A D \leq 4$ and $58.2 \%$ vs $66.7 \%$ at $A D \leq 7$. There were, for example, 28 vs 33 (Moura CG vs Ruppitsch CG) isolates matching with $A D=0$ to one of the 152 isolates in confirmed outbreaks, indicating that the real PPV and sensitivity may still be higher since all of these isolates would also have been classified as true positives.

\section{Microbiological cluster detection}

The proportion of sporadic cases, i.e. cases that do not cluster with any other, was $47.0 \%$ vs $47.8 \%$ using $A D \leq 7$ and $54.5 \%$ vs $55.2 \%$ using $A D \leq 4$. Figure $2 C$ shows the effect of sampling fraction on the proportion of sporadic cases, using rarefaction and the Moura CG (similar results for Ruppitsch CG, data not shown). The isolates that clustered were further stratified by belonging to either a single- or a multi-country cluster. Table 3 further stratifies the number of clusters based on size, cluster cutoff and single- vs multi-country. Among the multi-country clusters, a distinction is made between clusters in which each country has only one isolate, and that can thus only be detected at EU/EEA level, and clusters in which at least one country has more than one isolate. The latter can be detected first either at national level or at EU/EEA level, depending on whether the second isolate of any involved country is also the second isolate overall. The average number of days that EU/EEA level detection for clusters of more than five isolates was earlier than national detection was at least $75(A D \leq 4)$ and at most $154(A D \leq 7)$. This indicates that, in terms of timeliness of detection, there can be a substantial gain by pooling data at the EU/EEA level.

Finally, the 6-year time span of this dataset also allows examination of the evolution of clusters over time. Figure $2 \mathrm{~d}$ shows the aggregated number of isolates over a period of nearly 4 years belonging to the same cluster, with the first isolate in the cluster set at $\mathrm{t}=\mathrm{o}$. These clusters were created using single linkage clustering and different $A D$ cutoffs of $0, \leq 4$ and $\leq 7$ to assess the impact of the cutoff on cluster duration and size. For our dataset, and using cutoff $A D \leq 7$, clusters of sizes two, three and larger than three had a median duration of 16,78 and 112 weeks, respectively, for single-country clusters. For multi-country clusters, this increased to 34,84 and 181 weeks, respectively.

\section{Discussion}

In this large multi-country study, we examined both the analytical and general epidemiological aspects of surveillance for human $\mathrm{Lm}$ infections using WGS. We identified CGC as the one main quality indicator that detected most of the quality issues in WGS data. CGM and contamination checks were also investigated, but these identified few additional issues, although further research on the extent and impact of contaminations and mixed cultures is warranted. The low occurrence of contamination indicates a high level of quality among participating laboratories. Quality control criteria were established to classify isolates as 'Accepted', 'AcceptedForOutbreak' and 'Rejected'.

We conclude that the isolate sequences classified as 'Accepted' by the criteria outlined here are of sufficient quality to be a reliable basis for supporting epidemiological investigations and, in particular, cluster detection and outbreak investigations, also in an international setting. Although $2.3 \%$ of the isolates did not reach this level of quality, there is a clear correlation with the average coverage up to around $55 \mathrm{x}$ before trimming and $45 \mathrm{x}$ after trimming for the Illumina platform. This would therefore be a recommended minimum coverage for this platform for laboratories getting started with WGS for $L m$ to ensure the reliability of the data and downstream analyses, consistent with previous reports [10]. It is also possible that further improvements through, for example, internal and external quality assessment exercises and new sequencing technology, could result in lower coverage yielding the same quality.

Based on the comparison of different allele calling methods, it can be concluded that assembly-based allele calling outperforms reads-based allele calling with the methods used here. More loci were detected, which increased typeability, and the average distances between isolates were slightly smaller. Velvet including k-mer optimisation performed slightly worse than SPAdes, but both produced near-equivalent results. This provides an important opportunity to simplify the cooperation between national public health institutes and laboratories on WGS-enhanced surveillance, thereby enhancing their ability to respond to listeriosis cross-border clusters and outbreaks. Provided that validated assemblers are used to assure data reproducibility [10], and in the absence of a globally accepted unique strain nomenclature to classify isolates, it is sufficient for cgMLST analysis to share assembled genomes rather than sequence read data, which often require an additional separate system and workload. When further confirmation is required, e.g. when deciding on control measures in multi-country outbreaks, it may still be necessary to share sequence reads, e.g. for SNP analysis or to verify the analyses, but this is likely only for a small minority of the cases. For communication on outbreaks or detected clusters, it is important to keep in mind that the individual differences in ADs between Moura and Ruppitsch CG schemes can be relatively large, due to the different set of loci used, since only 1,261 loci are common to both schemes [10]. This may impact the formulation of outbreak case definitions, which should always specify what cgMLST scheme(s) are used, as well as their total number of loci, minimum WGS quality criteria and cutoffs. 
The $A D \leq 7$ cutoff defined in Moura et al. is confirmed as useful for cluster detection for both the Moura and the Ruppitsch CG $[10,23]$. A more stringent cluster cutoff of $A D \leq 4$ in combination with single linkage clustering may be considered for identifying isolates with more compelling microbiological evidence of being part of the same outbreak. A second higher cutoff for weaker microbiological evidence could be applied as well, in order to define, for example, confirmed vs probable cases in an outbreak case definition. More confirmed outbreaks are needed to have a more accurate estimation of PPV and sensitivity as a function of cluster cutoff. Since confirmed outbreaks were delineated at national level only and using less discriminatory methods than cgMLST, there may be additional isolates in the dataset that are actually part of one of the confirmed outbreaks, leading to underestimation of PPV and possibly sensitivity. More confirmed outbreaks are also needed to understand why epidemiologically linked isolates could have substantially more allele differences than the cutoff, as described earlier $[9,10]$. One possibility for microbiological diversity in an epidemiological cluster is that a single source or environmental niche may be occupied by more than one strain. Another is that specific sublineages may have higher average mutation rates, and if sufficient evidence is available to justify it, sublineage-specific cutoffs could be defined. Reliability of establishing microbiological relatedness is crucial in practice for public health, since it increases the power of analytical studies on exposure data. In addition, though unlikely for $L m$ given its low incidence and high severity, when exposure data cannot be gathered for all cases, it would help in selecting the cases for which to gather exposure data.

The aggregated curves of number of cases as a function of time were also similar regardless of the cutoff used (Figure 1d), indicating that clusters of closely related isolates can persist for several years, as previously reported [11,26-28]. Such a long time span of microbiological clusters can be expected given the microbiological properties of $L m$, such as a slow mutation rate of around 1 SNP per year and the ability to form biofilms that are difficult to eradicate in food processing environments $[6,7,10,29]$. This is important to take into account, especially for control measures, since knowledge of retrospective isolates, whether from human cases or food sources, may still inform investigations of new cases $[9,11,27,30]$. Therefore, an additional restriction on time for defining microbiological clusters of $L m$ does not seem warranted for descriptive epidemiology and hypothesis generation, while it is likely still required for analytical epidemiological studies.

Around a third of the clusters found involved more than one country, and for clusters of more than five isolates this increased to around half. This can be expected given the international nature of food trade, and indicates that there is potential for a substantial added public health value of introducing EU/EEA-wide, WGS-enhanced surveillance of listeriosis. Among other benefits, it may lead to earlier detection of clusters, which in our dataset was on average several months for multi-country clusters. At the same time, the molecular typing results must also be combined with epidemiological and food exposure investigations. Given the sometimes-long incubation period of listeriosis, the low number of cases and the severity of the disease, food exposure data should ideally be collected for all cases, without additional waiting for typing results, as presently done in, for example, Denmark, France and the US. It should also be complemented by WGS typing of officially sampled food isolates, and be combined with a joint analysis of the microbiological data to detect potential links between human cases and food items [11,31]. The successful implementation of control measures for multi-country persistent clusters also requires cross-border investigations and intense collaboration of public health and food safety authorities and laboratories.

Assuming that all isolates clustering within the cutoff are epidemiologically linked, there is a substantial amount of apparently sporadic cases [32]. Based on the rarefaction analysis, around half of the cases in this study were sporadic cases. However, this proportion is likely to be slightly lower for comprehensive sampling. Any bias in the selection of isolates in the different countries, with respect to their likelihood of being part of an outbreak, may also affect this number. It is likely that some proportion of cases is truly sporadic in the sense that they are isolated cases related to individual- or household-level food preparation or storage practices, rather than to the microbiological quality of the food at the time of purchase [33]. In general, these cases can only be addressed through preventive measures such as public education, rather than control measures for food business operators. Further research is needed to determine to what extent specific populations face an increased risk, as well as how preventive measures such as microbiological criteria for food could be improved. Finally, similar studies could be useful for other pathogens, in particular Salmonella enterica, Shiga toxin-/verocytotoxin-producing Escherichia coli (STEC/VTEC) and Campylobacterspp., to establish appropriate sequence quality criteria, to assess epidemiological concordance as a function of the analysis method and to estimate the added value of national and EU/EEA-wide WGS-enhanced surveillance.

\section{European Listeria WGS typing group}

Wesley Mattheus (Wetenschappelijk Instituut
Volksgezondheid, Brussels, Belgium), Iva Christova (National
Center of Infectious and Parasitic Diseases, Sofia, Bulgaria),
Panayiota Maikanti-Charalampous (Nicosia General
Hospital, Nicosia, Cyprus), Renáta Karpíšková (Výzkumný
ústav veterinárního lékařství, Brno, Czech Republic), Sven
Halbedel (Robert Koch-Institut, Wernigerode, Germany),
Eva Møller Nielsen (Statens Serum Institut, Copenhagen,
Denmark), Marliin Koolmeister (Terviseamet, Tallinn,


Estonia), Georgia Mandilara (National Reference Center for Salmonella, Shigella, VTEC and Listeria, Vari Attiki, Greece), Raquel Abad Torreblanca (Instituto de Salud Carlos III, Madrid, Spain), Saara Salmenlinna (National Institute for Health and Welfare, Helsinki, Finland), Marc Lecuit (Institut Pasteur, National Reference Center and WHO Collaborating Center Listeria, Biology of Infection Unit, Inserm U1117, and Paris Descartes University, Paris, France), Alexandre Leclercq (Institut Pasteur, National Reference Center and WHO Collaborating Center Listeria, Paris, France), Ivelina Damjanova (Országos Epidemiológia Központ, Budapest, Hungary), Niall Delappe (University Hospital Galway, Galway, Ireland), Guðrún Sigmundsdóttir (Landspítali University Hospital, Reykjavik, Iceland), Antonietta Gattuso (Instituto Superiore di Sanità, Rome, Italy), Algirdas Griškevičius (National Public Health Surveillance Laboratory, Vilnius, Lithuania), Catherine Ragimbeau (Laboratoire national de santé, Dudelange, Luxembourg), Eelco Franz (Rijksinstituut voor Volksgezondheid en Milieu, Bilthoven, Netherlands), Lin Thorstensen Brandal (Norwegian Institute of Public Health, Oslo, Norway), Alicja Kuch (National Medicines Institute, Warsaw, Poland), Vítor Borges (Instituto Nacional de Saúde Doutor Ricardo Jorge, Lisbon, Portugal), Dana Caplan (Cantacuzino National Institute for Research, Bucharest, Romania), Cecilia Jernberg (The Public Health Agency of Sweden, Stockholm, Sweden), Erik Alm (The Public Health Agency of Sweden, Stockholm, Sweden), Marija Trkov (National Laboratory of Health, Environment and Food, Ljubljana, Slovenia), Eva Tkacova (National Reference Laboratory for Listeriosis, Kosice, Slovakia), Kathie Grant (Public Health England, London, United Kingdom).

\section{Acknowledgements}

We thank Alfonsina Fiore (Istituto Superiore di Sanità, Italy), Jani Halkilahti (National Institute for Health and Welfare, Finland) and Judit Pászti (Országos Epidemiológia Központ, Hungary) for technical support. Daniel Palm (ECDC, Sweden), Joana Revez (ECDC), Marc Struelens (ECDC), Max Heck (Rijksinstituut voor Volksgezondheid en Milieu, Netherlands), Mike Catchpole (ECDC) and Saara Kotila (ECDC) for critical review. Marc Struelens (ECDC) and Sylvain Brisse (Institut Pasteur, France) for advice and guidance.

\section{Funding:}

Timothy Dallman (Public Health England (PHE), United Kingdom): The research in the United Kingdom was funded by the National Institute for Health Research Health Protection Research Unit (NIHR HPRU) in Gastrointestinal Infections at University of Liverpool in partnership with Public Health England, in collaboration with University of East Anglia, University of Oxford and the Institute of Food Research.

The views expressed are those of the author(s) and not necessarily those of the National Health Service (NHS), the NIHR, the Department of Health or PHE.

Alexandra Moura, Alexandre Leclercq and Marc Lecuit: The research in France was supported by Institut Pasteur, Inserm and Santé Publique France.

\section{Conflict of interest}

None declared.

\section{Authors' contributions}

Ivo Van Walle: lead author, conception and design, drafting, intellectual input, data collection, analyses. Jonas Torgny Björkman: intellectual input, critical review. Martin Cormican: intellectual input, critical review. Timothy Dallman: intellectual input, critical review, analyses. Joël Mossong: intellectual input, critical review, analyses. Alexandra Moura: intellectual input, critical review, analyses. Ariane Pietzka: intellectual input, critical review. Werner Ruppitsch: intellectual input, critical review, analyses. European Listeria WGS typing group: intellectual input, critical review, data acquisition. Johanna Takkinen: conception and design, intellectual input, critical review. All authors read and approved the final version of the manuscript.

\section{References}

1. de Noordhout CM, Devleesschauwer B, Angulo FJ, Verbeke G, Haagsma J, Kirk M, et al. The global burden of listeriosis: a systematic review and meta-analysis. Lancet Infect Dis. 2014;14(11):1073-82. https://doi.org/10.1016/S14733099(14)70870-9 PMID: 25241232

2. Charlier C, Perrodeau É, Leclercq A, Cazenave B, Pilmis B, Henry $B$, et al. MONALISA study group. Clinical features and prognostic factors of listeriosis: the MONALISA national prospective cohort study. Lancet Infect Dis. 2017;17(5):5109. https://doi.org/10.1016/S1473-3099(16)30521-7 PMID: 28139432

3. Lamont RF, Sobel J, Mazaki-Tovi S, Kusanovic JP, Vaisbuch E, Kim SK, et al. Listeriosis in human pregnancy: a systematic review. J Perinat Med. 2011;39(3):227-36. https://doi. org/10.1515/jpm.2011.035 PMID: 21517700

4. European Centre for Disease Prevention and Control (ECDC) Surveillance Atlas of Infectious Diseases. Stockholm: ECDC. [Accessed: 10 Mar 2016]. Available from: http://atlas.ecdc. europa.eu

5. Goulet V, King LA, Vaillant V, de Valk H. What is the incubation period for listeriosis? BMC Infect Dis. 2013;13(1):11. https:// doi.org/10.1186/1471-2334-13-11 PMID: 23305174

6. Lundén J, Autio T, Markkula A, Hellström S, Korkeala H. Adaptive and cross-adaptive responses of persistent and nonpersistent Listeria monocytogenes strains to disinfectants. Int J Food Microbiol. 2003;82(3):265-72. https://doi.org/10.1016/ S0168-1605(02)00312-4 PMID: 12593929

7. Keto-Timonen R, Tolvanen R, Lundén J, Korkeala H. An 8-year surveillance of the diversity and persistence of Listeria monocytogenes in a chilled food processing plant analyzed by amplified fragment length polymorphism. J Food Prot. 2007;70(8):1866-73. https://doi.org/10.4315/0362028X-70.8.1866 PMID: 17803143

8. Gerner-Smidt P, Hise K, Kincaid J, Hunter S, Rolando S, HyytiäTrees E, et al. Pulsenet Taskforce. PulseNet USA: a five-year update. Foodborne Pathog Dis. 2006;3(1):9-19. https://doi. org/10.1089/fpd.2006.3.9 PMID: 16602975

9. Jackson BR, Tarr C, Strain E, Jackson KA, Conrad A, Carleton H, et al. Implementation of Nationwide Real-time Whole-genome Sequencing to Enhance Listeriosis Outbreak Detection and Investigation. Clin Infect Dis. 2016;63(3):380-6. https://doi. org/10.1093/cid/ciw242 PMID: 27090985

10. Moura A, Criscuolo A, Pouseele H, Maury MM, Leclercq A, Tarr C, et al. Whole genome-based population biology and epidemiological surveillance of Listeria monocytogenes. Nat Microbiol. 2016;2(2):16185. https://doi.org/10.1038/ nmicrobiol.2016.185 PMID: 27723724

11. Moura A, Tourdjman M, Leclercq A, Hamelin E, Laurent E, Fredriksen N, et al. Real-Time Whole-Genome Sequencing for Surveillance of Listeria monocytogenes, France. Emerg Infect Dis. 2017;23(9):1462-70. https://doi.org/10.3201/ eid2309.170336 PMID: 28643628

12. European Centre for Disease Prevention and Control (ECDC) ECDC roadmap for integration of molecular and genomic typing into European-level surveillance and epidemic preparedness - Version 2.1, 2016-2019. Stockholm: ECDC; 2016. Available from: http://ecdc.europa.eu/en/publications/Publications/ molecular-typing-EU-surveillance-epidemic-preparedness2016-19-roadmap.pdf

13. van Belkum A, Tassios PT, Dijkshoorn L, Haeggman S, Cookson B, Fry NK, et al. European Society of Clinical Microbiology and Infectious Diseases (ESCMID) Study Group on Epidemiological Markers (ESGEM). Guidelines for the validation and application of typing methods for use in bacterial epidemiology. Clin Microbiol Infect. 2007;13(Suppl 3):1-46. https://doi. org/10.1111/j.1469-0691.2007.01786.x PMID: 17716294

14. Mellmann A, Harmsen D, Cummings CA, Zentz EB, Leopold SR, Rico A, et al. Prospective genomic characterization of the German enterohemorrhagic Escherichia coli $0_{104}: \mathrm{H}_{4}$ outbreak by rapid next generation sequencing technology. 
PLoS One. 2011;6(7):e22751. https://doi.org/10.1371/journal. pone.0022751 PMID: 21799941

15. Maiden MC, Jansen van Rensburg MJ, Bray JE, Earle SG, Ford SA, Jolley KA, et al. MLST revisited: the gene-by-gene approach to bacterial genomics. Nat Rev Microbiol. 2013;11(10):728-36. https://doi.org/10.1038/nrmicro3093 PMID: 23979428

16. European Centre for Disease Prevention and Control (ECDC). Expert Opinion on the introduction of next-generation typing methods for food- and waterborne diseases in the EU and EEA. Stockholm: ECDC; 2015. Available from: https://ecdc.europa. eu/en/publications-data/expert-opinion-introduction-nextgeneration-typing-methods-food-and-waterborne

17. Nadon C, Van Walle I, Gerner-Smidt P, Campos J, Chinen I, Concepcion-Acevedo J, et al. FWD-NEXT Expert Panel. PulseNet International: Vision for the implementation of whole genome sequencing (WGS) for global food-borne disease surveillance. Euro Surveill. 2017;22(23):30544. https://doi. org/10.2807/1560-7917.ES.2017.22.23.30544 PMID: 28662764

18. Bolger AM, Lohse M, Usadel B. Trimmomatic: a flexible trimmer for Illumina sequence data. Bioinformatics. 2014;30(15):211420. https://doi.org/10.1093/bioinformatics/btu170 PMID: 24695404

19. Bankevich A, Nurk S, Antipov D, Gurevich AA, Dvorkin M, Kulikov AS, et al. SPAdes: a new genome assembly algorithm and its applications to single-cell sequencing. J Comput Biol. 2012;19(5):455-77. https://doi.org/10.1089/cmb.2012.0021 PMID: 22506599

20. Zerbino DR, Birney E. Velvet: algorithms for de novo short read assembly using de Bruijn graphs. Genome Res. 2008;18(5):821-9. https://doi.org/10.1101/gr.074492.107 PMID: 18349386

21. Li H, Durbin R. Fast and accurate long-read alignment with Burrows-Wheeler transform. Bioinformatics. 2010;26(5):58995. https://doi.org/10.1093/bioinformatics/btp698 PMID: 20080505

22. Langmead B, Salzberg SL. Fast gapped-read alignment with Bowtie 2. Nat Methods. 2012;9(4):357-9. https://doi. org/10.1038/nmeth.1923 PMID: 22388286

23. Ruppitsch W, Pietzka A, Prior K, Bletz S, Fernandez HL, Allerberger F, et al. Defining and evaluating a core genome multilocus sequence typing scheme for whole-genome sequence-based typing of Listeria monocytogenes. J Clin Microbiol. 2015;53(9):2869-76. https://doi.org/10.1128/ JCM.01193-15 PMID: 26135865

24. Altschul SF, Gish W, Miller W, Myers EW, Lipman DJ. Basic local alignment search tool. J Mol Biol. 1990;215(3):403-10. https:// doi.org/10.1016/So022-2836(05)80360-2 PMID: 2231712

25. Maury MM, Tsai YH, Charlier C, Touchon M, Chenal-Francisque $\mathrm{V}$, Leclercq $\mathrm{A}$, et al. Uncovering Listeria monocytogenes hypervirulence by harnessing its biodiversity. Nat Genet. 2016;48(3):308-13. https://doi.org/10.1038/ng.3501 PMID: 26829754

26. Holch A, Webb K, Lukjancenko O, Ussery D, Rosenthal BM, Gram L. Genome sequencing identifies two nearly unchanged strains of persistent Listeria monocytogenes isolated at two different fish processing plants sampled 6 years apart. Appl Environ Microbiol. 2013;79(9):2944-51. https://doi. org/10.1128/AEM.03715-12 PMID: 23435887

27. Schmid D, Allerberger F, Huhulescu S, Pietzka A, Amar C, Kleta $S$, et al. Whole genome sequencing as a tool to investigate a cluster of seven cases of listeriosis in Austria and Germany, 2011-2013. Clin Microbiol Infect. 2014;20(5):431-6. https://doi. org/10.1111/1469-0691.12638 PMID: 24698214

28. Stasiewicz MJ, Oliver HF, Wiedmann M, den Bakker HC. Whole-Genome Sequencing Allows for Improved Identification of Persistent Listeria monocytogenes in Food-Associated Environments. Appl Environ Microbiol. 2015;81(17):6024-37. https://doi.org/10.1128/AEM.01049-15 PMID: 26116683

29. Orsi RH, Borowsky ML, Lauer P, Young SK, Nusbaum C, Galagan JE, et al. Short-term genome evolution of Listeria monocytogenes in a non-controlled environment. BMC Genomics. 2008;9(1):539. https://doi.org/10.1186/1471-21649-539 PMID: 19014550

30. Gillesberg Lassen S, Ethelberg S, Björkman JT, Jensen T, Sørensen G, Kvistholm Jensen A, et al. Two listeria outbreaks caused by smoked fish consumption-using whole-genome sequencing for outbreak investigations. Clin Microbiol Infect. 2016;22(7):620-4. https://doi.org/10.1016/j.cmi.2016.04.017 PMID: 27145209

31. European Collaborative Projects (Euroreference). The ECDCEFSA molecular typing database for European Union public health protection. Europe: Euroreference; 2017;2:4-12. Available from: https://euroreference.anses.fr/sites/default/ files $/ 17 \% 2003 \% 20$ ED\%20ER\%2002\%201_RIZZI.pdf

32. Field N, Cohen T, Struelens MJ, Palm D, Cookson B, Glynn JR, et al. Strengthening the Reporting of Molecular Epidemiology for
Infectious Diseases (STROME-ID): an extension of the STROBE statement. Lancet Infect Dis. 2014;14(4):341-52. https://doi. org/10.1016/S1473-3099(13)70324-4 PMID: 24631223

33. EFSA BIOHAZ Panel (EFSA Panel on Biological Hazards), Ricci A, Allende A, Bolton D, Chemaly M, Davies R, et al. Scientific opinion on the Listeria monocytogenes contamination of ready-to-eat foods and the risk for human health in the EU. EFSA Journal 2018;16(1):5134. Available from: https://efsa. onlinelibrary.wiley.com/doi/abs/10.2903/j.efsa.2018.5134

\section{License and copyright}

This is an open-access article distributed under the terms of the Creative Commons Attribution (CC BY 4.0) Licence. You may share and adapt the material, but must give appropriate credit to the source, provide a link to the licence, and indicate if changes were made.

This article is copyright of the authors, 2018. 